\title{
Defects in polynucleotide phosphorylase impairs virulence in Escherichia coli 0157:H7
}

\author{
Jia $H u^{1,2}$ and Mei-Jun Zhu ${ }^{1 *}$ \\ ${ }^{1}$ School of Food Science, Washington State University, Pullman, WA, USA, ${ }^{2}$ Department of Animal Science, University of \\ Wyoming, Laramie, WY, USA
}

\section{OPEN ACCESS}

Edited by:

Dongsheng Zhou,

Beijing Institute of Microbiology and Epidemiology, China

Reviewed by:

Yufeng Yao,

Shanghai Jiao Tong University School of Medicine, China

Grzegorz Wegrzyn,

University of Gdansk, Poland

*Correspondence:

Mei-Jun Zhu,

School of Food Science, Washington State University, Pullman, WA 99164

USA

meijun.zhu@wsu.edu

Specialty section:

This article was submitted to

Food Microbiology,

a section of the journal

Frontiers in Microbiology

Received: 31 May 2015

Accepted: 22 July 2015

Published: 17 August 2015

Citation:

Hu J and Zhu M-J (2015) Defects

in polynucleotide phosphorylase

impairs virulence in Escherichia coli

O157:H7.

Front. Microbiol. 6:806. doi: 10.3389/fmicb.2015.00806
Polynucleotide phosphorylase (PNPase) is reported to regulate virulence in Salmonella, Yersinia sp. and Campylobacter jejuni, yet its role in Escherichia coli 0157:H7 has not been investigated. To gain insights into its roles in E. coli $0157: \mathrm{H} 7$ virulence, pnp deletion mutants were generated and the major virulence factors were compared to their parental wild type strains. Deletion of pnp in E. coli O157:H7 dramatically decreased stx2 mRNA expression and Stx2 protein production, and impaired lambdoid prophage activation in E. coli O157:H7. Quantitative PCR further confirmed that the Stx2 phage lytic growth was repressed by pnp deletion. Consistent with reduced Stx2 production and Stx2 phage activation, the transcriptional levels of genes involved in phage lysis and replication were down-regulated. In addition, disruption of pnp in E. coli O157:H7 decreased its adhesion to intestinal epithelial cells as well as cattle colonic explant tissues. On the other hand, PNPase inactivation in E. coli O157:H7 enhanced Tir protein content and the transcription of type three secretion system components, including genes encoding intimin, Tir, and EspB as well as locus of enterocyte and effacement positive regulator, Ler. Collectively, data indicate that PNPase has pleiotropic effects on the virulence of $E$. coli $\mathrm{O} 157: \mathrm{H} 7$.

Keywords: E. coli 0157:H7, PNPase, Shiga toxin 2, prophage, Type three secretion system, intestine, epithelium, adhesion

\section{Introduction}

Shiga toxin (Stx) producing Escherichia coli O157:H7 is a major food safety threat that results in significant economic losses, especially in the beef industry. Stx is the major virulence factor in E. coli O157:H7, which causes bloody diarrhea and life threatening hemolytic-uremic syndrome (Mainil and Daube, 2005). The mortality associated with E. coli O157:H7 infection is due to the production and release of Stx, which is composed of a single 32-kDa A subunit and five 7.7-kDa B subunits (Paton and Paton, 1998). Stx binds to receptors on cell surface and is internalized through endocytosis, which inhibits protein synthesis and causes complications associated with $E$. coli O157:H7 infection (Johannes and Romer, 2010). In addition, E. coli O157:H7 has a chromosomal pathogenicity island, i.e., locus of enterocyte and effacement (LEE; McDaniel et al., 1995), which mediates intimate contact to epithelial cells, further leading to attaching and effacing (AE) lesions (McDaniel et al., 1995). The LEE is composed of five major operons (LEE1-5) that encode type three secretion system (T3SS) apparatus and effector proteins (Deng et al., 2004). 
There is a plethora of studies on the regulation of LEE in E. coli $\mathrm{O} 157: \mathrm{H7}$, which is regulated by multiple proteins such as Ler, H-NS, GrlA, CsrA through direct or indirect interaction (Bhatt et al., 2011). Compared with LEE, the regulation of Stx 2 production in E. coli $\mathrm{O} 157: \mathrm{H} 7$ has only been sparsely studied. Gene stx2 is localized on Stx2 prophage, a lambdoid like bacteriophage, DNA that inserted to E. coli O157:H7 genome (Herold et al., 2004; Allison, 2007; Los et al., 2011). Stx production is generally linked to the expression of induced Stx-phage's late genes, which results in phage lytic cycle induction and release of Stx (Wagner et al., 2001). The life cycle of prophages is controlled by phage repressor CI proteins in lambdoid phage (Ptashne and Hopkins, 1968). Mutations in the $c$ I gene make a non-cleavable repressor and an uninducible 933W Stx2 prophage (Tyler et al., 2013). RecA can stimulate the self-cleavage of CI further allowing initiation of transcription from the early $P_{\mathrm{L}}$ and $P_{\mathrm{R}}$ promoters (Little, 2005). The transcription from $P_{\mathrm{L}}$ results in expression of $N$ protein, which modified RNA polymerase initiating at $P_{\mathrm{L}}$ and $P_{\mathrm{R}}$ to form resistant to downstream terminators (Friedman $\mathrm{Di}, 2006)$. The transcription from $P_{\mathrm{R}}$ results in $\mathrm{Q}$ expression, which initiated the transcription from $P_{\mathrm{R}}{ }^{\prime}$ and the downstream genes expression including stx $A$ and $B$ (Karch et al., 1999). SOS response due to DNA damage and replication arrest can enhance $r e c A$ expression and further stimulate Stx production (Kimmitt et al., 2000). There is also a RecA independent lambdoid prophage activation pathway for Stx production in E. coli O157:H7 (Imamovic and Muniesa, 2012), which is through regulation of RcsA (Rozanov et al., 1998). However, the mechanism involved in RecA-independent Stx2 933W prophage induction remains unknown (Imamovic and Muniesa, 2012). Stx prophage is also regulated by poly (A) polymerase I (PAP I), and PAP I-deficient cells showed a significant impairment of lysogenization and lytic development by Stx phages (Nowicki et al., 2015).

Polynucleotide phosphorylase (PNPase) has both $3^{\prime}$ $5^{\prime}$ exoribonuclease activity and $3^{\prime}$ terminal oligonucleotide polymerase activity, which is involved in mRNA degradation and small RNA turnover (Carpousis et al., 1999; Arraiano et al., 2010) and also serves as PAP II (Kushner, 2015). PNPase regulates T3SS expression in Yersinia sp. and Salmonella Typhimurium (Ygberg et al., 2006; Rosenzweig et al., 2007); acts as a global regulator of virulence genes in Salmonella, which repressed invasion and intracellular replication of Salmonella (Clements et al., 2002), but was required for $S$. Typhimurium gut colonization in swine (Bearson et al., 2013). It enhanced Campylobacter jejuni motility and its chicken gut colonization (Haddad et al., 2012). However, roles of PNPase in the colonization and virulence of E. coli O157:H7 had not been explored, which were examined in the current study.

\section{Materials and Methods}

\section{Cell Line, Media, Bacterial Strains, and Plasmids}

The human colonic epithelial cell line HT-29 was obtained from the American Type Culture Collection (ATCC ${ }^{\circledR}$ HTB- $38^{\mathrm{TM}}$,
Manassas, VA, USA). HT-29 cells were routinely cultured in Dulbecco's Modified Eagle's medium (DMEM; Sigma, St. Louis, MO, USA) supplemented with $10 \%$ fetal bovine serum (Sigma), 100 units $/ \mathrm{ml}$ penicillin $\mathrm{G}$, and $100 \mu \mathrm{g} / \mathrm{ml}$ of streptomycin (Sigma).

The E. coli O157:H7 EDL933, Sakai, and 86-24 strains were obtained from the STEC center at Michigan State University. E. coli O157:H7 was routinely grown in $\mathrm{LB}$ broth at $37^{\circ} \mathrm{C}$ with aeration. E. coli O157:H7 pnp deletion mutant was generated per the published method (Datsenko and Wanner, 2000). Briefly, using the forward primer GGCTTTACCCACATAGAGCTGGGTT AGGGTTGTCATTAGTCGCGAGGATGattccggggatccgtcgacc, and the reverse primer: CCGCCGCAGCGGAYGGCAAATGGC AACCTTACTCGCCCTGTTCAGCAGC tgtaggctggagctgcttcg, Kanamycin resistance cassette (lowercase letter) with $p n p$ homology flanking sequence (uppercase letters) was PCRamplified from pKD13 and electroporated into E. coli O157:H7 containing the $\lambda$-Red recombinase plasmid. The deletion of $p n p$ was confirmed by PCR amplification using primers external to the disrupted gene (forward primer: TGTCA TTAGTCGCGAGGATG; and reverse primer: GCGGAYGGCA AATGGCAACC). To construct the pnp complementary plasmid, the pnp gene was PCR-amplified from E. coli O157:H7 genomic DNA using primers flanking with KpnI and $X b a \mathrm{I}$ restriction enzyme sites, respectively (underlined letters): ATGGTACCACGCAAACGACACCGGTTCT and AG TCTAGATTACTCGCCCTGTTCAGCAG, and cloned into pBBR1-MCS3 (Kovach et al., 1995), which was kindly provided by Dr. Mark Gomelsky. The resulting plasmid pBBR1MCS3::pnp and its control vector were electroporated into E. coli O157:H7 EDL933 pnp mutant strains, which resulted in six strains: (1) EDL933; (2) EDL933 $\Delta p n p$; (3) EDL933 $\Delta p n p$ pBBR1; (4) EDL933 $\Delta p n p$ pBBR1::pnp; (5) 86-24 $\Delta p n p$; (6) Sakai $\Delta p n p$. Given that $p n p$ and its downstream genes, $y h b M$ or nlpI (Lipoprotein NlpI), deaD (ATP-dependent RNA helicase) and $m t r$ (tryptophan permease) are located in one operon, to confirm that pnp deletion did not impact transcription of its downstream genes, we further compared the transcription of $y h b M$, the nearest downstream gene of pnp, between pnp deletion mutant and wild-type strain using forward primer: CAGTTTGAACAGTGCCGTGG and reverse primer: GATAACACCTCGCTCGCTGA.

\section{Immunoblotting}

Supernatant components: overnight E. coli O157:H7 cultures were subcultured at $1: 1,000$ in $\mathrm{LB}$ broth for $14 \mathrm{~h}$ at $37^{\circ} \mathrm{C}$ under shaking at $200 \mathrm{rpm}$, when bacteria were in stationary phase. Bacterial cultures were centrifuged at 5,000 $\times g$ at room temperature for $5 \mathrm{~min}$, and the supernatant was then filtered through $0.22 \mu \mathrm{m}$ filter (Millipore, Bedford, MA, USA). Trichloroacetic acid was added to a final concentration of $10 \%$ to the supernatant and incubated overnight at $4^{\circ} \mathrm{C}$ to precipitate proteins.

Whole bacteria: overnight $E$. coli $\mathrm{O} 157: \mathrm{H} 7$ cultures were subcultured at 1:1,000 in $\mathrm{LB}$ broth for $14 \mathrm{~h}$ at $37^{\circ} \mathrm{C}$ under shaking at $200 \mathrm{rpm}$, when bacterial culture were used for protein extraction. 
Protein extracts were separated by $10 \%$ SDS-PAGE, transferred to nitrocellulose membranes and assayed with antibodies specific to Stx2A (Toxin Technology Inc., Sarasota, FL, USA) and Tir (a generous gift from Dr. John Leong, Tufts University). Blotted membranes were visualized using $\mathrm{ECL}^{\mathrm{TM}}$ Western blotting detection reagents (Amersham Bioscience, Piscataway, NJ, USA).

\section{Phage Spontaneous Induction and Enumeration}

Phage enumeration assay was conducted according to our published method (Harris et al., 2012; Yue et al., 2012). Briefly, E. coli $\mathrm{O} 157$ cells were activated from frozen glycerol stock and grown in $\mathrm{LB}$ broth at $37^{\circ} \mathrm{C}$ with aeration for $8 \mathrm{~h}$, which were sub-cultured at $1: 1,000$ in $\mathrm{LB}$ broth for $14 \mathrm{~h}$ at $37^{\circ} \mathrm{C}$ under shaking at $200 \mathrm{rpm}$ when cultures were used to assess phage spontaneous induction. Activated bacterial culture was centrifuged at $5,000 \times g$ at $4^{\circ} \mathrm{C}$ for $5 \mathrm{~min}$. The resulting supernatant was serially diluted in a phage solution containing $10 \mathrm{mM} \mathrm{CaCl}_{2}$ (Sigma) and $5 \mathrm{mM} \mathrm{MgSO}_{4}$ (Sigma). Proper phage titration $(200 \mu \mathrm{l})$ was mixed with $0.9 \mathrm{ml}$ of the sensitive E. coli strain MG1655 culture and $5 \mathrm{ml}$ of tempered top LB agar $(0.7 \%, \mathrm{~W} / \mathrm{V})$ supplemented with $10 \mathrm{mM} \mathrm{CaCl}_{2}$ and $10 \mathrm{mM}$ $\mathrm{MgSO}_{4}$. They were then mixed and poured immediately on top of the bottom LB agar plates $(1.5 \%, \mathrm{~W} / \mathrm{V})$ containing $3 \mu \mathrm{g} / \mathrm{ml}$ chloramphenicol (Sigma). Plates were incubated at $37^{\circ} \mathrm{C}$ and plaque forming units (Pfu) were counted after $36 \mathrm{~h}$ incubation. Measurements for each strain had four independent replicates.

\section{Quantitative PCR (qPCR) Analysis of Stx2 Prophage}

Shiga toxin2 prophage qPCR was conducted per published methods (Harris et al., 2012; Yue et al., 2012). Briefly, overnight bacterial cultures were centrifuged at $5,000 \times g$ at $4^{\circ} \mathrm{C}$ for $5 \mathrm{~min}$. The supernatant was then filtered through $0.22 \mu \mathrm{m}$ filter (Millipore, Bedford, MA, USA) to ensure complete removal of bacteria. The filtrate was centrifuged at $35,000 \times g$ at $4^{\circ} \mathrm{C}$ for $2 \mathrm{~h}$. The resulting phage pellet was dissolved in sterile $\mathrm{ddH}_{2} \mathrm{O}$ and treated with DNase I (2 units/ $\left.\mu \mathrm{l}\right)$ at $37^{\circ} \mathrm{C}$ for $2 \mathrm{~h}$ to hydrolyze any remaining contaminated E. coli O157:H7 genomic DNA. The above phage preparation was boiled at $100^{\circ} \mathrm{C}$ for $10 \mathrm{~min}$ to denature DNase I and release phage DNA, and then used as a template for qPCR. PCR analysis of stx2 gene was conducted using stx $2 A$ primers and SYBR Green master mix (Bio-Rad, Hercules, CA, USA). Absence of bacterial DNA in phage lysates was confirmed by the absence of tufA DNA as assayed by PCR. Stx2 phages are known to carry only one copy of the stx 2 gene, therefore the stx2 gene copy number can be extrapolated to quantify Stx2 prophages.

\section{Adhesion of E. coli 0157:H7 to Colonic Epithelial Cells}

HT-29 epithelial cells were seeded in 24 well plates and cultured in DMEM media (Sigma) with $10 \%$ FBS (Sigma) until $\sim 90$ $\%$ confluence. The medium was removed and each well was washed three times with PBS ( $\mathrm{pH}$ 7.4). The cells in each well were challenged with $10^{7} \mathrm{CFU} /$ well E. coli O157:H7. The HT-29 cells and $E$. coli $\mathrm{O} 157: \mathrm{H} 7$ were co-cultured at $37^{\circ} \mathrm{C}$ with $5 \% \mathrm{CO}_{2}$ for $2 \mathrm{~h}$. Then the HT-29 cell monolayers were washed three times with PBS, and lysed with $0.2 \%$ Triton X-100. Lysates were serially diluted, plated on LB, and bacterial colonies were counted after $18 \mathrm{~h}$ incubation (Xicohtencatl-Cortes et al., 2007; Torres et al., 2008).

\section{E. coli 0157:H7 Adhesion to Cattle Colonic Explants}

Terminal colons (5-10 cm from anus) were aseptically collected from E. coli O157:H7 free beef cattle slaughtered in the University of Wyoming Meat Laboratory and transferred to the Microbiology Lab within $10 \mathrm{~min}$. Specimens were washed five times with $0.9 \%(\mathrm{~W} / \mathrm{V}) \mathrm{NaCl}$ and the mucosa was dissected from the underlying tissues using sterile dissecting scissors. The trimmed specimens were reduced to $8 \mathrm{~mm}$ round pieces using Miltex disposable biopsy punches (Cardinal Health, Cleveland, $\mathrm{OH}$, USA), and transferred to the individual well of 24 well plates and cultured in DMEM medium with 5\% FBS for $1 \mathrm{~h}$. E. coli $\mathrm{O} 157: \mathrm{H} 7$ was added to these wells at $10^{7} \mathrm{CFU} /$ well, and cultured at $37^{\circ} \mathrm{C}$ for $2 \mathrm{~h}$ (Xicohtencatl-Cortes et al., 2007). The attached E. coli O157:H7 cells were serially diluted, plated and enumerated.

\section{Quantitative Reverse Transcription PCR (qRT-PCR) Analyses}

Total RNA was extracted from E. coli O157:H7 grown in LB broth using RNeasy Protect Bacteria Mini Kit (Qiagen, Valencia, CA, USA) and reverse transcribed using a QuantiTect Reverse Transcription Kit (Qiagen). cDNAs were used as a template for qRT-PCR analysis of selected genes using a CFX96 ${ }^{\mathrm{TM}}$ Real-Time PCR Detection System (Bio-Rad, Hercules, CA, USA). SYBR Green Master Mix (Bio-Rad, Hercules, CA, USA) was used for all qRT-PCR reactions. Primers for qRT-PCR are listed in Tables $\mathbf{1}$ and 2. Gene gapA was used as the housekeeping gene. Amplification efficiency was $0.90-0.99$.

\section{Statistical Analysis}

Data were analyzed as a randomized design using GLM (General Linear Model of Statistical Analysis System, SAS, 2000). Differences between means were determined using Student's $t$-test followed by Duncan's multiple test when appropriate. $P<0.05$ was considered to be statistically significant.

\section{Results}

\section{PNPase is Essential for Stx2 Production in E. coli 0157:H7 EDL933 Strain}

In transcriptional level, deletion of $p n p$ completely eliminated stx2 mRNA expression in E. coli O157:H7 EDL933 strain without affecting stx1 expression (Figure 1A). We further compared the Stx 2 production. In wild-type strain, a $32 \mathrm{kDa}$ band Stx 2 A subunit was detected in both supernatant fraction and 
TABLE 1 | Primer sets used for quantitative Reverse Transcription PCR (qRT-PCR) for Shiga toxin (Stx) and phage related genes.

\begin{tabular}{|c|c|c|c|}
\hline Gene name & Product size & Direction & Sequence \\
\hline \multirow[t]{2}{*}{$\mathrm{cl}$} & $80 \mathrm{bp}$ & Forward & TCACACGAAGACCAAAGGCA \\
\hline & & Reverse & TGCCATCGAGATGACCGAAG \\
\hline \multirow[t]{2}{*}{$c l l$} & $118 \mathrm{bp}$ & Forward & АCCTGTCAACGCTTACCCAG \\
\hline & & Reverse & GATGCCATGCCAAAAGCACA \\
\hline \multirow[t]{2}{*}{ clll } & $101 \mathrm{bp}$ & Forward & TTCTITGGGACTCCTGGCTG \\
\hline & & Reverse & GGCTGCCCTTCCGAATCTIT \\
\hline \multirow[t]{2}{*}{ cro } & $75 \mathrm{bp}$ & Forward & GAAGTTGCGAAGGCTTGTGG \\
\hline & & Reverse & AGTCTTAGGGAGGAAGCCGT \\
\hline \multirow[t]{2}{*}{ gapA } & $103 \mathrm{bp}$ & Forward & CCAGGACATCGTTTCCAAC \\
\hline & & Reverse & GGTGGTCATCAGACCTTCG \\
\hline \multirow[t]{2}{*}{0} & $104 \mathrm{bp}$ & Forward & GATGCTGCAATTCAGAGCGG \\
\hline & & Reverse & TाTCTGGCTGATGGTGCGAT \\
\hline \multirow[t]{2}{*}{$\operatorname{rcs} A$} & $124 \mathrm{bp}$ & Forward & CTCGACGATATCCTTGGCGA \\
\hline & & Reverse & ССТGACCTGCCATCCACATT \\
\hline \multirow[t]{2}{*}{ rec $A$} & $70 \mathrm{bp}$ & Forward & CTGGGAGCACAGCAGGTTGTCG \\
\hline & & Reverse & TGAACACGCGCTGGACCCAATC \\
\hline \multirow[t]{2}{*}{$S$} & $124 \mathrm{bp}$ & Forward & AGTTGCTGGACAGGGTTTCC \\
\hline & & Reverse & GCCTTACGCCGGTCTTCTाT \\
\hline \multirow[t]{2}{*}{ stx1 } & $180 \mathrm{bp}$ & Forward & ATAAATCGCCATTCGTTGACTAC \\
\hline & & Reverse & AGAACGCCCACTGAGATCATC \\
\hline \multirow[t]{2}{*}{ stx2A } & 133 bp & Forward & CGTCACTCACTGGTTTCATCAT \\
\hline & & Reverse & TCTGTATCTGCCTGAAGCGTAA \\
\hline
\end{tabular}

TABLE 2 | Primer sets used for qRT-PCR for T3SS related genes.

\begin{tabular}{|c|c|c|c|}
\hline Gene name & Product size & Direction & Sequence \\
\hline \multirow[t]{2}{*}{ csrA } & 102 bp & Forward & TCCTTCGGGGCATTTACGCCA \\
\hline & & Reverse & TCGTCGAGTTGGTGAGACCCTC \\
\hline \multirow[t]{2}{*}{ eae } & $139 \mathrm{bp}$ & Forward & TCACTTTGAATGGTAAAGGCAGT \\
\hline & & Reverse & CAAATGGACATAGCATCAGCATA \\
\hline \multirow[t]{2}{*}{ espB } & $122 \mathrm{bp}$ & Forward & TAAAGGGGCTGGTGAGATTG \\
\hline & & Reverse & CTGCGACATCAGCAACACTT \\
\hline \multirow[t]{2}{*}{ gapA } & $103 \mathrm{bp}$ & Forward & CCAGGACATCGTTCCAAC \\
\hline & & Reverse & GGTGGTCATCAGACCTTCG \\
\hline \multirow[t]{2}{*}{$\operatorname{grl} / A$} & $71 \mathrm{bp}$ & Forward & TAGAAAGTCCTGGAACAAC \\
\hline & & Reverse & AGACTGTCCCACAATACC \\
\hline \multirow[t]{2}{*}{ hns } & $150 \mathrm{bp}$ & Forward & GCCGGACGCTGAGCACGTT \\
\hline & & Reverse & CGCGGCTGCTGCTGAAGTTG \\
\hline \multirow[t]{2}{*}{ ler } & $71 \mathrm{bp}$ & Forward & CCCGACCAGGTCTGCCCTTCT \\
\hline & & Reverse & GATGGACTCGCTCGCCGGAAC \\
\hline \multirow[t]{2}{*}{ tir } & $128 \mathrm{bp}$ & Forward & AACGAAAGAAGCGTTCCAGA \\
\hline & & Reverse & СTGCTGCTTAAGCCTGCTCT \\
\hline
\end{tabular}

whole cell lysis (Figure 1B). Deletion of $p n p$ resulted in no detection of Stx2A (Figure 1B). This defective in Stx2 protein production was not restored by complementation of pBBR1::pnp (Figure 1B).

\section{PNPase is Indispensable for Stx2 Phage Lytic Growth in E. coli O157:H7}

Shiga toxin production is mediated by Stx2 phage activation, thus we further compared phage activation in E. coli O157:H7 EDL933 and its $p n p$ mutant strains. Deletion of $p n p$ completely abolished lambdoid phage progeny production in E. coli O157:H7 (Figure 2A). Quantitative PCR further confirmed that the stx2 phage DNA accumulation was repressed in PNPase defective E. coli O157:H7 strain (Figure 2B). To gain insights into molecular mechanisms of PNPase mediated impairment in Stx phage activation, we further analyzed expression of phage genes regulating prophage activation. Deletion of $p n p$ did not affect recA and rcsA expressions (Figure 2C). We could not detect cro mRNA expression and only detected a very low level of $c$ I expression in $\Delta p n p$ strain (Figure 2C). In addition, the transcriptional levels of $c \mathrm{II}$ and $c \mathrm{III}$, phage lysis $S$ gene and phage replication gene $O$ were all diminished in $\Delta p n p$ strain (Figure 2C). Altogether, these data indicated that PNPase in E. coli $\mathrm{O} 157: \mathrm{H} 7$ plays an indispensable role in Stx2 prophage lysis.

\section{Deletion of PNPase in E. coli O157:H7 Impairs Epithelial Adhesion but Enhances T3SS Encoding Transcripts and Proteins}

In addition to phage encoding Stx2 production, E. coli O157:H7 has a LEE chromosomal pathogenicity island that encoding T3SS apparatus and effectors, mediating intimate contact to epithelial cells (McDaniel et al., 1995). We further evaluated the significance of $p n p$ deletion in E. coli O157: H7 epithelial adhesion. Deletion of pnp in E. coli O157: H7 EDL933 strain decreased its adhesion to colonic epithelial HT-29 cells by approximately twofold (Figure 3A). Consistently, adhesion to cattle colonic gut explant was reduced about fourfold in EDL933 $\Delta p n p$ (Figure 3B).

To understand possible factors limiting E. coli O157:H7 epithelial colonization in the absence of PNPase, we further analyzed T3SS apparatus and effectors transcripts and proteins in EDL933 $\Delta p n p$ and its wild type strains. Surprisingly, the transcriptional levels of genes located on LEE region including tir, eae, and $e s p B$ were enhanced in EDL933 $\triangle p n p$ strain (Figure 4A). Tir protein production was also increased in both supernatant fraction and whole cell lysis of EDL933 $\Delta p n p$ strain compared to the wild-type EDL933 strain (Figure 4B). In line with, the transcriptional level of LEE positive regulators, the ler transcript level, was significantly enhanced in EDL933 $\Delta p n p$ strain (Figure 4C), though other regulators of LEE ( $h n s, c s r A$, and grlA) did not differ between EDL933 $\Delta p n p$ and its isogenic wild type strain.

\section{Impact of PNPase in Other E. coli 0157:H7 Strains}

To confirm the results from E. coli O157:H7 strain EDL933, we further examined the Stx2 production and Stx2 phage lytic activation in E. coli O157:H7 strain Sakai and 86-24. Consistent to the finding in EDL933 strain, the pnp deletion decreased Stx2 production (Figure 5A) and impaired Stx2 phage lytic growth (Figure 5B) in Sakai and 86-24. As in strain EDL933, Tir protein content was enhanced in both E. coli O157:H7 strain Sakai and 86-24 (Figure 5A). 
A

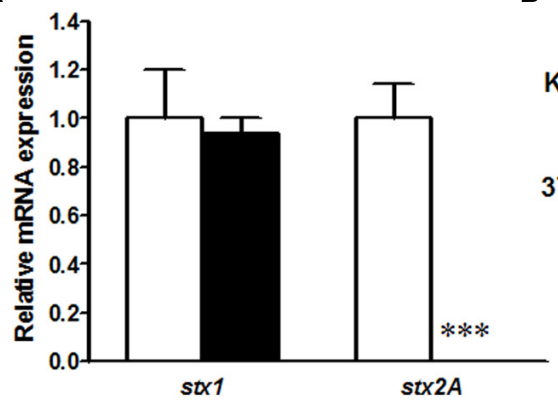

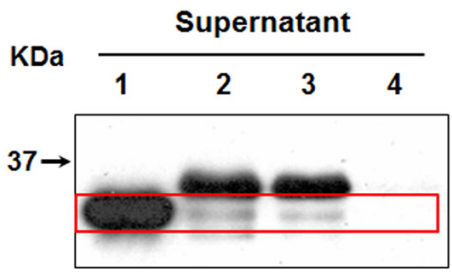

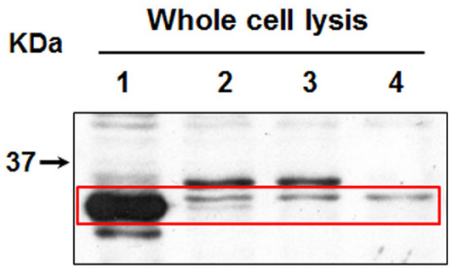

1: EDL933; 2: EDL933Apnp; 3: pBBR1; 4: pBBR1::pnp
FIGURE 1 | Shiga toxin production in E. coli 0157:H7 EDL933 strains. (A) Relative mRNA expression; (B) Relative protein content. EDL933: wild-type E. coli O157:H7 strain; EDL933 $\Delta$ pnp: EDL933 pnp deletion mutant strain;
pBBR1: EDL933 $\Delta p n p$ strain carrying an empty vector pBBR1; pBBR1::pnp: EDL933 $\Delta$ pnp strain complemented with pBBR1::pnp. $\square$ : EDL933; EDL933 $\Delta$ pnp; *** $P<0.001$ (Mean \pm SEM; $n=4$ ).
A

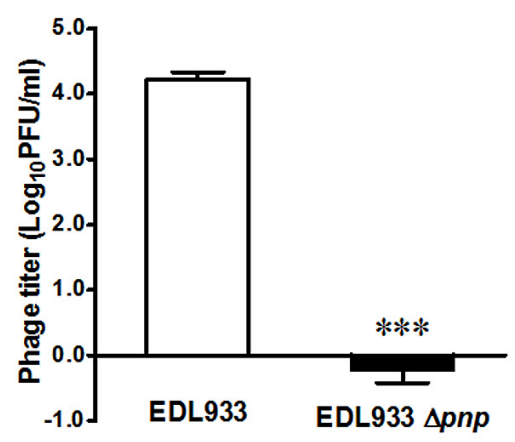

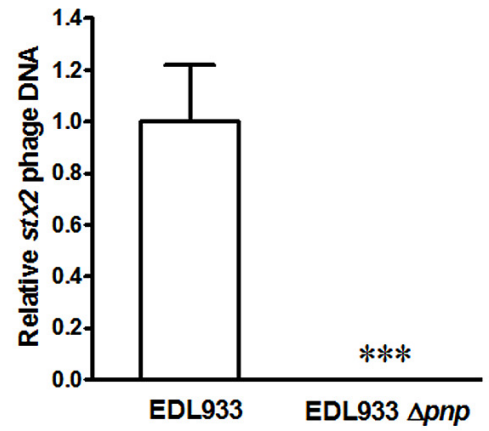

C

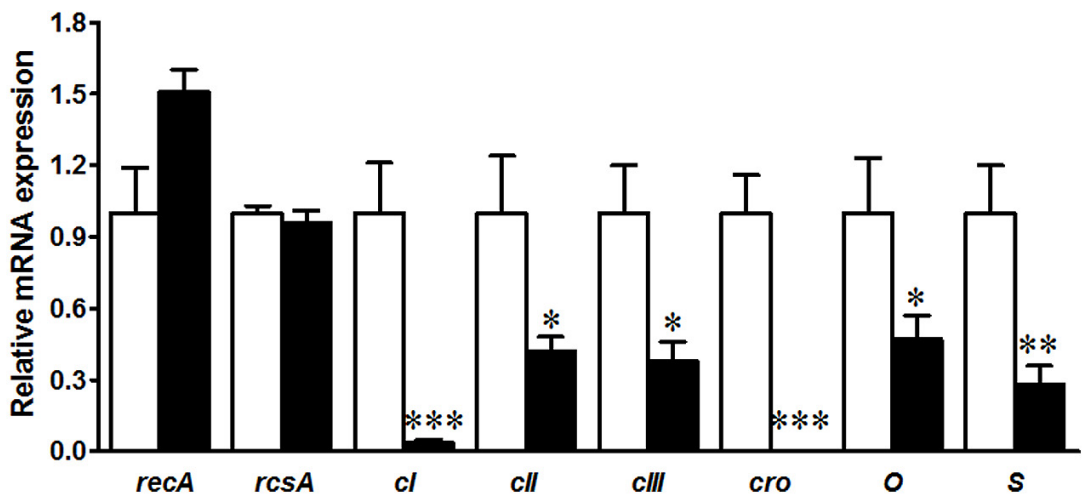

FIGURE 2 | Prophage enumeration in E. coli 0157:H7 EDL933 wild type ( $\square$ ) and pnp deletion strains ( $\square$ ). (A) Spontaneous lambdoid prophage enumeration; (B) Stx2 phage PCR; (C) Phage lysis related gene expressions. ${ }^{* *} P<0.001,{ }^{*} P<0.01,{ }^{*} P<0.05$ (Mean \pm SEM, $n=4$ ).

\section{Discussion}

\section{PNPase Regulates Stx2 Production Through Repressing Stx2 Phage Lytic Cycle}

Shiga toxin is mainly responsible for the mortality associated with E. coli O157:H7 infection. PNPase is a bifunctional enzyme with an exoribonuclease activity and a poly (A) polymerase activity. Study herein demonstrated that PNPase plays a vital role in Stx production. The deletion of $p n p$ in E. coli $\mathrm{O} 157: \mathrm{H} 7$ completely eliminated stx 2 mRNA expression and Stx2 production, which could not be recovered by complementary pnp overexpression. Thus we speculated that the observed effect of PNPase on Stx2 production in EDL933 strain might be due to a secondary mutation in stx2 gene during generation of $p n p$ deletion mutant. Therefore, we did three independent $p n p$ deletion experiments. Different colony from three independent knock out experiments had the same Stx2 profile (data not shown). We further sequenced the promoter region of $s t x 2$, which revealed no alteration in the sequence of stx2 promoter region (data not shown). Given 
A

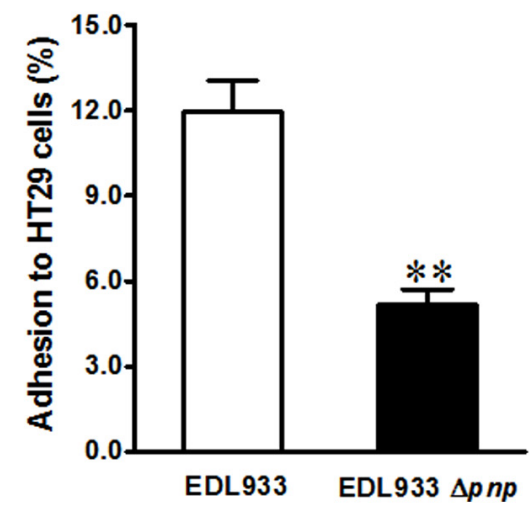

B

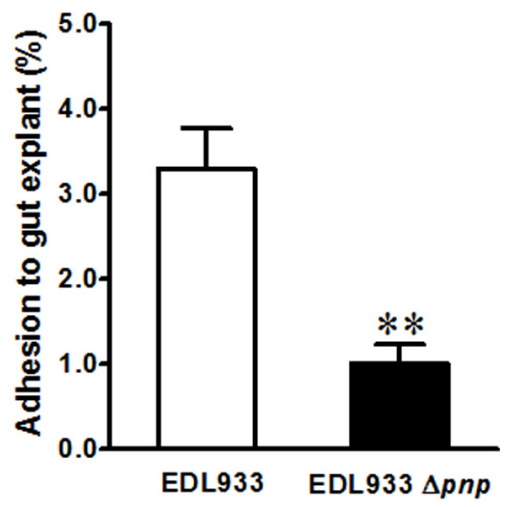

FIGURE 3 | Adhesion of E. coli O157:H7 to intestinal epithelium. (A) HT-29 colonic epithelial cell line; (B) Cattle colonic explant tissues. EDL933: wild-type E. coli O157:H7 strain; EDL933 $\Delta$ pnp: EDL933 pnp deletion mutant strain, ${ }^{* *} P<0.01$ (Mean $\pm \mathrm{SEM} ; n=8$ ).

that $p n p$ and its downstream genes, $y h b M$, deaD, and $m t r$ are in one operon, we questioned whether disruption of $p n p$ might change the mRNA levels of pnp downstream genes. Thus, we further analyzed the transcription of the pnp nearest downstream gene, $y h b M$, and qRT-PCR analysis indicated that it was not affected by pnp deletion (data not shown). These testimonies showed that the decreased Stx2 production was due to $p n p$ deletion. Although the polar effect of $p n p$ deletion would be low in our experiment (Our experiment is in frame deletion), we can't rule out the possibility that the polar effect of the pnp gene deletion cause the deficiency of Stx production.

Effective production of Stx requires phage lytic status development. Consistent with decreased Stx production, pnp deletion in E. coli O157:H7 repressed Stx2 phage lytic status, indicating that the lytic growth of $933 \mathrm{w}$ phage in E. coli O157:H7 was regulated by PNPase. CI protein is required for lysogenic establishment and has self-feedback inhibition activity, which represses prophage replication and lysis (Echols and Green, 1971). cro is the early lytic gene antagonizes CI and regulates phage switch from lysogenic to lytic growth during prophage induction (Svenningsen et al., 2005). Consistent with impaired Stx2 phage lytic growth, transcriptional level of cro were decreased in the EDL933 $\Delta$ pnp strain. Additionally, transcriptional levels of $c \mathrm{II}$ and $c \mathrm{III}$, phage lysis gene, $S$, and replication genes, $O$, were also repressed in $\Delta p n p$ compared to the wild type host. These data demonstrated that the lytic cycle of the Stx 2 phage in EDL933 $\Delta p n p$ strain was repressed, which at least partially through influencing transcripts of gene encoding proteins vital for lytic phage growth. In line with our findings, dysfunction of PAP I in E. coli resulted in impaired Stx phage lytic development associated with reduced transcripts of cII, cIII, cro, and $O$ at later time points of induction (Nowicki et al., 2015). Of note, the transcript level of $O$ and cro are altered differently in $\Delta p n p$ relative to wild-type strain, which might be due to the different post-transcriptional regulation (e.g., different sRNA regulation) and warrants further research.
Similar to what observed in strain EDL933, deletion of pnp significantly decreased Stx2 production in Sakai and 8624 strains. EDL933 and Sakai strains have both stx1 and stx2 genes, while $86-24$ only has stx2 gene. $933 \mathrm{~W}$ prophage from EDL933 and Sakai Stx2 prophage have different sequence in early gene regulators and replication proteins (Makino et al., 1999), but almost identical sequence for structural genes (Makino et al., 1999). Compared to the genome of 86-24 Stx2 producing phage, there is a 1439 bp insertion replaced a 2091 bp sequence in $933 \mathrm{~W}$ phage (Kudva et al., 2002). In agreement with diminished Stx 2 production, Stx 2 phage spontaneous lytic growth was repressed in all tested PNPase deficient strains, indicating that PNPase is important in lytic cycle development of Stx2 phage in E. coli O157:H7 strains.

\section{PNPase Reduced E. coli 0157:H7 Intestinal Epithelial Colonization Independent of Expression Levels of T3SS}

Deletion of pnp in E. coli O157:H7 decreased its adhesion to epithelial cells and colonic epithelial tissues. In agreement, C. jejun deficient in PNPase has reduced ability to adhere and invade to HT-29 cells, and colonize chick gut (Haddad et al., 2012). PNPase was required for S. Typhimurium gut colonization in swine, PNPase deficiency was associated with reduced fecal $S$. Typhimurium shedding and intestinal colonization (Bearson et al., 2013). These data collectively indicated PNPase plays a critical role in gut colonization.

Escherichia coli O157:H7 possess LEE pathogenicity island that encode T3SS apparatus and effector proteins, enabling its intimate contact to epithelial cells and further forming AE lesions. In contrast to decreased epithelial adhesion, we revealed that $p n p$ deletion elevated the transcription level of genes located in the LEE region as well as Tir protein content, which is possibly through enhancing ler transcription. These data indicated that PNPase in E. coli O157:H7 might regulate expression and functional activity 


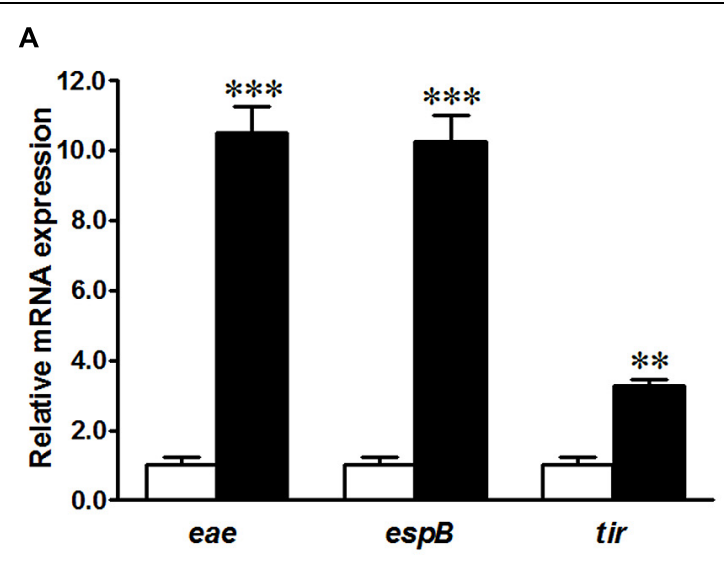

8

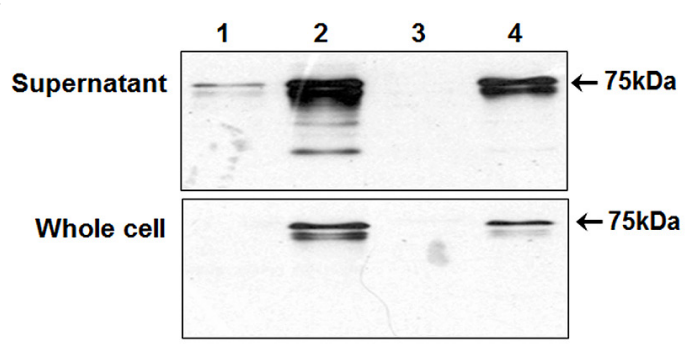

1: EDL933; 2: EDL933 Apnp; 3: pBBR1::pnp; 4: pBBR1

C

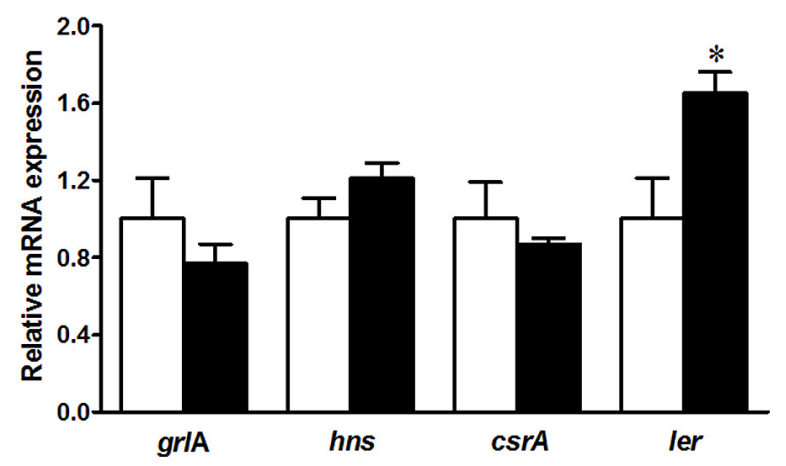

FIGURE 4 | Type three secretion system (T3SS) and its regulators in E. coli 0157:H7 EDL933 strains. (A) mRNA expression of T3SS; (B) Tir protein content in E. coli O157:H7; (C) mRNA expressions of T3SS regulators. EDL933: wild-type E. coli O157:H7 strain; EDL933 $\Delta$ pnp: EDL933 pnp deletion mutant strain; pBBR1: EDL933 $\Delta p n p$ strain carrying an empty vector pBBR1; pBBR1::pnp: EDL933 $\Delta$ pnp strain complemented with pBBR1::pnp. $\square$ : EDL933; $\mathbf{\square}$ : EDL933 $\Delta$ pnp; ${ }^{* *} P<0.001,{ }^{* *} P<0.01,{ }^{*} P<0.05$ (Mean \pm SEM; $n=4$ ).

of T3SS independently, which warrants future research. The reduced T3SS functional activity as indicated by reduced epithelial adhesion might be due to the defectiveness in T3SS apparatus organization or effector secretion. Meanwhile, we speculated that the decreased intestinal adhesion might be partially due to the reduced Stx 2 production. Stx regulates the distribution of nucleolin, a host cell protein that is a receptor for intimin in E. coli O157:H7 (Robinson et al., 2006). PNPase deletion decreased Stx2, which might disturb nucleolin

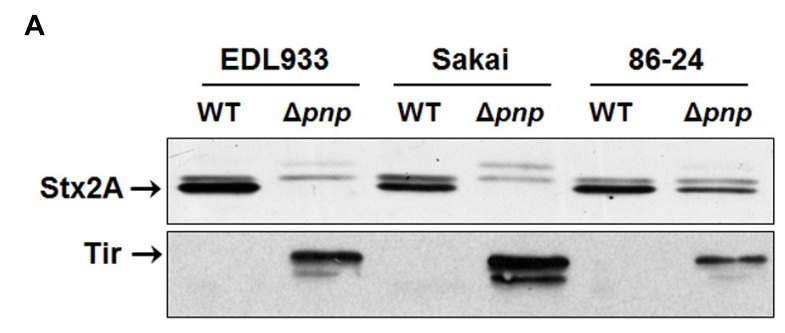

B

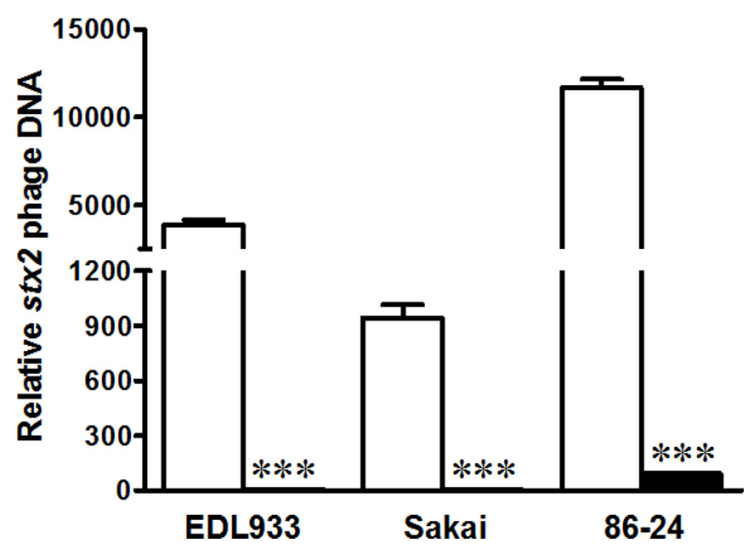

FIGURE 5 | Effects of PNPase on the virulence factors of $E$. coli 0157:H7 EDL933, Sakai, and 86-24 strains. (A) Stx $2 A$ and Tir protein contents by immunoblotting; (B) stx2 phage DNA content by qPCR. $\square$ : wild-type (WT); $\mathbf{\square}$ : pnp deletion mutant $(\Delta p n p)$. ${ }^{* * *} P<0.001$ (Mean $\pm \mathrm{SEM}$, $n=4)$.

distribution and further impair the adherence capacity of E. coli O157:H7.

Our observation was supported by previous studies in Yersinia, where PNPase is required for the proper T3SS function. The pnp mutant strain was defective in rapidly exporting T3SS effector proteins (Rosenzweig et al., 2005), less virulent in mouse, but enhanced T3SS encoding transcripts and proteins compared with its isogenic wild-type Yersinia strain (Rosenzweig et al., 2007). Similarly, in S. Typhimurium, despite of decreased swine intestinal colonization in response to PNPase deficiency (Bearson et al., 2013), T3SS-encoding transcripts and proteins were expressed at higher levels in PNPase inactivated strain (Ygberg et al., 2006). On the other hand, inactivation of PNPase in Salmonella increased invasion and intracellular replication as well as the establishment of persistent infection in mice (Clements et al., 2002). These implicated a complex nature of PNPases in regulating bacterial virulence.

In summary, PNPase has pleiotropic effects on the virulence of E. coli O157:H7. It is not only indispensable for Stx2 phage lytic cycle growth and associated Stx2 production in E. coli O157:H7, but also required for $E$. coli $\mathrm{O} 157: \mathrm{H} 7$ intestinal epithelial adhesion and regulation of type III secretion system. Alteration in PNPase activity might provide a potential strategy to reduce the virulence of E. coli O157:H7. 


\section{Acknowledgments}

We thank Dr. Xin Fang and Dr. Mark Gomelsky at Department of Molecular Biology, University of Wyoming for their helpful suggestions. We acknowledge Dr. John Leong at Tufts

\section{References}

Allison, H. E. (2007). Stx-phages: drivers and mediators of the evolution of STEC and STEC-like pathogens. Future Microbiol. 2, 165-174. doi: 10.2217/17460913.2.2.165

Arraiano, C. M., Andrade, J. M., Domingues, S., Guinote, I. B., Malecki, M., Matos, R. G., et al. (2010). The critical role of RNA processing and degradation in the control of gene expression. FEMS Microbiol. Rev. 34, 883-923. doi: 10.1111/j.1574-6976.2010.00242.x

Bearson, S. M., Bearson, B. L., Lee, I. S., and Kich, J. D. (2013). Polynucleotide phosphorylase (PNPase) is required for Salmonella enterica serovar Typhimurium colonization in swine. Microb. Pathog. 65, 63-66. doi: 10.1016/j.micpath.2013.10.001

Bhatt, S., Romeo, T., and Kalman, D. (2011). Honing the message: post transcriptional and post-translational control in attaching and effacing pathogens. Trends Microbiol. 19, 217-224. doi: 10.1016/j.tim.2011. 01.004

Carpousis, A. J., Vanzo, N. F., and Raynal, L. C. (1999). mRNA degradation. A tale of poly(A) and multiprotein machines. Trends Genet. 15, 24-28. doi 10.1016/S0168-9525(98)01627-8

Clements, M. O., Eriksson, S., Thompson, A., Lucchini, S., Hinton, J. C., Normark, S., et al. (2002). Polynucleotide phosphorylase is a global regulator of virulence and persistency in Salmonella enterica. Proc. Natl. Acad. Sci. U.S.A. 99, 8784-8789. doi: 10.1073/pnas.132047099

Datsenko, K. A., and Wanner, B. L. (2000). One-step inactivation of chromosomal genes in Escherichia coli K-12 using PCR products. Proc. Natl. Acad. Sci. U.S.A. 97, 6640-6645. doi: 10.1073/pnas.120163297

Deng, W., Puente, J. L., Gruenheid, S., Li, Y., Vallance, B. A., Vazquez, A., et al. (2004). Dissecting virulence: systematic and functional analyses of a pathogenicity island. Proc. Natl. Acad. Sci. U.S.A. 101, 3597-3602. doi: 10.1073/pnas.0400326101

Echols, H., and Green, L. (1971). Establishment and maintenance of repression by bacteriophage lambda: the role of the cI, cII, and c3 proteins. Proc. Natl. Acad. Sci. U.S.A. 68, 2190-2194. doi: 10.1073/pnas.68.9.2190

Friedman Di, C. D. (2006). "Regulation of lambda gene experssion by transcription termination and antitermination," in The Bacteriophages, ed. R. Calendar (Oxford: Oxford Press.), 83-103.

Haddad, N., Tresse, O., Rivoal, K., Chevret, D., Nonglaton, Q., Burns, C. M., et al. (2012). Polynucleotide phosphorylase has an impact on cell biology of Campylobacter jejuni. Front. Cell. Infect. Microbiol. 2:30. doi 10.3389/fcimb.2012.00030

Harris, S. M., Yue, W. F., Olsen, S. A., Hu, J., Means, W. J., Mccormick, R. J., et al. (2012). Salt at concentrations relevant to meat processing enhances Shiga toxin 2 production in Escherichia coli O157:H7. Int J. Food Microbiol. 159, 186-192. doi: 10.1016/j.ijfoodmicro.2012. 09.007

Herold, S., Karch, H., and Schmidt, H. (2004). Shiga toxin-encoding bacteriophages-genomes in motion. Int. J. Med. Microbiol. 294, 115-121. doi: 10.1016/j.ijmm.2004.06.023

Imamovic, L., and Muniesa, M. (2012). Characterizing RecA-independent induction of Shiga toxin2-encoding phages by EDTA treatment. PLOS ONE 7:e32393. doi: 10.1371/journal.pone.0032393

Johannes, L., and Romer, W. (2010). Shiga toxins-from cell biology to biomedical applications. Nat. Rev. Microbiol. 8, 105-116. doi: 10.1038/nrmicro2279

Karch, H., Schmidt, H., Janetzki-Mittmann, C., Scheef, J., and Kroger, M. (1999). Shiga toxins even when different are encoded at identical positions in the genomes of related temperate bacteriophages. Mol. Gen. Genet. 262, 600-607. doi: $10.1007 / \mathrm{s} 004380051122$

Kimmitt, P. T., Harwood, C. R., and Barer, M. R. (2000). Toxin gene expression by shiga toxin-producing Escherichia coli: the role of antibiotics and the
University and Dr. Mark Gomelsky at University of Wyoming for their generous gifts of Tir antibodies and pBBR1-MCS3 plasmid, respectively. This work was supported by USDA-AFRI 2010-65201-20599 and Washington State University Emerging Research Issues Competitive Grants.

bacterial SOS response. Emerg. Infect. Dis. 6, 458-465. doi: 10.3201/eid0605. 000503

Kovach, M. E., Elzer, P. H., Hill, D. S., Robertson, G. T., Farris, M. A., Roop R. M. II, et al. (1995). Four new derivatives of the broad-host-range cloning vector pBBR1MCS, carrying different antibiotic-resistance cassettes. Gene 166, 175-176. doi: 10.1016/0378-1119(95)00584-1

Kudva, I. T., Evans, P. S., Perna, N. T., Barrett, T. J., Ausubel, F. M., Blattner, F. R. et al. (2002). Strains of Escherichia coli O157:H7 differ primarily by insertions or deletions, not single-nucleotide polymorphisms. J. Bacteriol. 184, 1873-1879. doi: 10.1128/JB.184.7.1873-1879.2002

Kushner, S. R. (2015). Polyadenylation in E. coli: a 20 year odyssey. RNA 21, 673-674. doi: 10.1261/rna.049700.115

Little, J. (2005). "Lysogeny, prophage induction, and lysogenic conversion," in Phages: Their Role in Bacterial Pathogenesis and Biotechnology, eds D. I. Friedman, M. K. Waldor, and S. L. Adhya (Washington, DC: ASM Press), 37-54.

Los, J. M., Los, M., and Wegrzyn, G. (2011). Bacteriophages carrying Shiga toxin genes: genomic variations, detection and potential treatment of pathogenic bacteria. Future Microbiol. 6, 909-924. doi: 10.2217/fmb.11.70

Mainil, J. G., and Daube, G. (2005). Verotoxigenic Escherichia coli from animals, humans and foods: who's who? J. Appl. Microbiol. 98, 1332-1344. doi: 10.1111/j.1365-2672.2005.02653.x

Makino, K., Yokoyama, K., Kubota, Y., Yutsudo, C. H., Kimura, S., Kurokawa, K. et al. (1999). Complete nucleotide sequence of the prophage VT2-Sakai carrying the verotoxin 2 genes of the enterohemorrhagic Escherichia coli O157:H7 derived from the Sakai outbreak. Genes Genet. Syst. 74, 227-239. doi: 10.1266/ggs.74.227

McDaniel, T. K., Jarvis, K. G., Donnenberg, M. S., and Kaper, J. B. (1995). A genetic locus of enterocyte effacement conserved among diverse enterobacterial pathogens. Proc. Natl. Acad. Sci. U.S.A. 92, 1664-1668. doi: 10.1073/pnas.92.5.1664

Nowicki, D., Bloch, S., Nejman-Falenczyk, B., Szalewska-Palasz, A., Wegrzyn, A. and Wegrzyn, G. (2015). Defects in RNA polyadenylation impair both lysogenization by and lytic development of Shiga toxin-converting bacteriophages. J. Gen. Virol. doi: 10.1099/vir.0.000102 [Epub ahead of print].

Paton, J. C., and Paton, A. W. (1998). Pathogenesis and diagnosis of Shiga toxin-producing Escherichia coli infections. Clin. Microbiol. Rev. 11, 450-479.

Ptashne, M., and Hopkins, N. (1968). The operators controlled by the lambda phage repressor. Proc. Natl. Acad. Sci. U.S.A. 60, 1282-1287. doi $10.1073 /$ pnas.60.4.1282

Robinson, C. M., Sinclair, J. F., Smith, M. J., and O’Brien, A. D. (2006). Shiga toxin of enterohemorrhagic Escherichia coli type O157 : H7 promotes intestinal colonization. Proc. Natl. Acad. Sci. U.S.A. 103, 9667-9672. doi: $10.1073 /$ pnas.0602359103

Rosenzweig, J. A., Chromy, B., Echeverry, A., Yang, J., Adkins, B., Plano, G. V., et al. (2007). Polynucleotide phosphorylase independently controls virulence factor expression levels and export in Yersinia spp. FEMS Microbiol. Lett. 270, 255-264. doi: 10.1111/j.1574-6968.2007.00689.x

Rosenzweig, J. A., Weltman, G., Plano, G. V., and Schesser, K. (2005). Modulation of Yersinia type three secretion system by the $\mathrm{S} 1$ domain of polynucleotide phosphorylase. J. Biol. Chem. 280, 156-163. doi: 10.1074/jbc.M405662200

Rozanov, D. V., D’Ari, R., and Sineoky, S. P. (1998). RecA-independent pathways of lambdoid prophage induction in Escherichia coli. J. Bacteriol. 180, 6306-6315.

Svenningsen, S. L., Costantino, N., Court, D. L., and Adhya, S. (2005). On the role of Cro in lambda prophage induction. Proc. Natl. Acad. Sci. U.S.A. 102, 4465-4469. doi: 10.1073/pnas.0409839102

Torres, A. G., Slater, T. M., Patel, S. D., Popov, V. L., and Arenas-Hernandez, M. M. (2008). Contribution of the Ler- and H-NS-regulated long polar fimbriae of 
Escherichia coli O157:H7 during binding to tissue-cultured cells. Infect. Immun. 76, 5062-5071. doi: 10.1128/IAI.00654-08

Tyler, J. S., Beeri, K., Reynolds, J. L., Alteri, C. J., Skinner, K. G., Friedman, J. H., et al. (2013). Prophage induction is enhanced and required for renal disease and lethality in an EHEC mouse model. PLoS Pathog. 9:e1003236. doi: 10.1371/journal.ppat.1003236

Wagner, P. L., Neely, M. N., Zhang, X., Acheson, D. W., Waldor, M. K., and Friedman, D. I. (2001). Role for a phage promoter in Shiga toxin 2 expression from a pathogenic Escherichia coli strain. J. Bacteriol. 183, 2081-2085. doi: 10.1128/JB.183.6.2081-2085.2001

Xicohtencatl-Cortes, J., Monteiro-Neto, V., Ledesma, M. A., Jordan, D. M., Francetic, O., Kaper, J. B., et al. (2007). Intestinal adherence associated with type IV pili of enterohemorrhagic Escherichia coli O157:H7. J. Clin. Invest. 117, 3519-3529. doi: 10.1172/JCI 30727

Ygberg, S. E., Clements, M. O., Rytkonen, A., Thompson, A., Holden, D. W., Hinton, J. C., et al. (2006). Polynucleotide phosphorylase negatively controls spv virulence gene expression in Salmonella enterica. Infect. Immun. 74, 1243-1254. doi: 10.1128/IAI.74.2.1243-1254.2006

Yue, W. F., Du, M., and Zhu, M. J. (2012). High temperature in combination with UV irradiation enhances horizontal transfer of stx2 gene from E. coli O157:H7 to non-pathogenic E. coli. PLoS ONE 7:e31308. doi: 10.1371/journal.pone. 0031308

Conflict of Interest Statement: The authors declare that the research was conducted in the absence of any commercial or financial relationships that could be construed as a potential conflict of interest.

Copyright $(2015 \mathrm{Hu}$ and Zhu. This is an open-access article distributed under the terms of the Creative Commons Attribution License (CC BY). The use, distribution or reproduction in other forums is permitted, provided the original author(s) or licensor are credited and that the original publication in this journal is cited, in accordance with accepted academic practice. No use, distribution or reproduction is permitted which does not comply with these terms. 